\title{
New approach to the calculation of the refractive index of liquid and solid xenon
}

\author{
Akira Hitachi \\ Kochi Medical School, Nankoku, Kochi 783-8505, Japan, and Laboratory for Instrumentation and Particle \\ Physics (LIP-Coimbra), Physics Department, University of Coimbra, 3004-516 Coimbra, Portugal
}

Vitaly Chepel, ${ }^{a)}$ M. Isabel Lopes, and Vladimir N. Solovov

Laboratory for Instrumentation and Particle Physics (LIP-Coimbra), Physics Department, University of Coimbra, 3004-516 Coimbra, Portugal

(Received 29 July 2005; accepted 11 October 2005; published online 19 December 2005)

\begin{abstract}
A model for the calculation of the refractive index based on a dispersion relation that incorporates the exciton transition energies is presented. The refractive indices of liquid and solid xenon for different wavelengths are evaluated using this model. Comparison between calculated and experimental values existing in the literature is presented. () 2005 American Institute of Physics.
\end{abstract}

[DOI: $10.1063 / 1.2136879]$

\section{INTRODUCTION}

The refractive index of liquid xenon is of interest both from the fundamental and application points of view. Presently, its practical relevance is mainly related to the use of liquid xenon in radiation detectors such as in particle physics and astrophysics, as well as its use in medical applications. Detailed information on this issue can be found in Refs. 1 and 2 and references therein. Many of those detectors rely on the measurement of scintillation light emitted by xenon when excited by a particle. There is, therefore, a strong interest in knowing the refractive index of liquid xenon at the wavelength corresponding to its scintillation light.

Liquid xenon emits in the vacuum ultraviolet region (vuv). Its emission spectrum is centered ${ }^{3}$ at $\lambda=178 \mathrm{~nm}$ and has a width of about $14 \mathrm{~nm}$. The emission originates ${ }^{4}$ from the transition from excimer (self-trapped exciton) states $\left({ }^{1} \Sigma_{u}^{+}\right.$ and ${ }^{3} \Sigma_{u}^{+}$) to the repulsive ground state ${ }^{1} \Sigma_{g}^{+}$. Accurate measurements of the optical constants for the scintillation light are difficult, mainly because of the short wavelength and low temperature. To our knowledge, there are very few published measurements of the refractive index of liquid xenon at the wavelength corresponding to xenon scintillation at present. A value of 1.66 was obtained in Ref. 5 for $\lambda=177 \mathrm{~nm}$ at the temperature slightly above the triple point, $163.2 \mathrm{~K}$. At the triple point, $n=1.5655 \pm 0.0024$ was reported for liquid for xenon scintillation light. ${ }^{6}$ In our recent measurements ${ }^{7}$ we obtained a refractive index of $1.69 \pm 0.02$ at the liquid temperature of $170 \mathrm{~K}$, also for the scintillation light.

Concerning the theoretical point of view, the precise knowledge of the refractive index is remarkably important, among the optical properties, not only by itself but also because it has been used in evaluating the success of theoretical models and the accurate calculation of other properties, namely, the density and polarizability of nonpolar fluids, by means of the Lorentz-Lorenz equation

${ }^{a)}$ Electronic mail: vitaly@lipc.fis.uc.pt

$$
\frac{n^{2}-1}{n^{2}+2} \frac{1}{\rho}=\frac{4 \pi}{3} \frac{N_{A}}{M} \alpha
$$

where $n$ is the refractive index of a homogeneous medium, $\rho$ is the density, $\alpha$ is the molecular polarizability, $M$ is the molecular weight, and $N_{A}$ is Avogadro's number. The quantity at the left side of the equation, is known as the LorentzLorenz function ${ }^{8,9} F_{\mathrm{LL}}$

$$
F_{\mathrm{LL}}=\frac{n^{2}-1}{n^{2}+2} \frac{1}{\rho} \text {. }
$$

Condensed rare gases are ideal media to examine the theory. Their atoms interact by means of weak van der Waals forces; the overlapping of the electron charge distributions is very small and, therefore, the local electric field can be approximated with good accuracy by the Lorentz field equal to $\boldsymbol{E}+(4 \pi / 3) \boldsymbol{P}$, where $\boldsymbol{E}$ is the macroscopic average electric field in the medium and $\boldsymbol{P}$ is the total polarization of the medium. It was also shown that the Lorentz field is quite accurate for most of the solids with face-centered-cubic lattice, which is the case of solid Ar, Kr, and Xe (see Ref. 10).

In a system of noninteracting atoms, $F_{\mathrm{LL}}$ is independent of the density as one can see from Eqs. (1) and (2). However, in a high-pressure gas or condensed medium such dependence can appear. Indeed, variations of $F_{\mathrm{LL}}$ with density were reported in Refs. 11 and 12. For the static case, with $n^{2}=\varepsilon$, a similar behavior was also observed for the ClausiusMossotti function ${ }^{13,14} F_{\mathrm{CM}}$

$$
F_{\mathrm{CM}}=\frac{\varepsilon-1}{\varepsilon+2} \frac{1}{\rho} \text {. }
$$

In order to describe this phenomenon theoretically, $F_{\mathrm{LL}}$ and $F_{\mathrm{CM}}$ were fitted by using virial expansion, in powers of the density ${ }^{15,16} \rho$. To explain the density effect, a number of corrections were also suggested, such as introducing fluctuations in the induced molecular dipole momentum, ${ }^{17}$ change in polarizability due to the caging of individual molecules by their neighbors at higher density, ${ }^{18}$ and quadrupole- 
quadrupole and higher-order interactions. ${ }^{19}$ Contribution from two-body ${ }^{16}$ or many-body ${ }^{20}$ interactions to the effective polarizability has also been considered. In addition, the order of the media can be an important factor affecting the refractive index. The solids may have reduced $F_{\mathrm{LL}}$ compared with liquid due to the high symmetry of the crystal lattice. ${ }^{9}$ However, the variations in $F_{\text {LL }}$ with density and phase are very small, such that the spread of data on $\rho(T)$ existing in the literature renders difficult the comparison of calculations of $F_{\text {LL }}$ done by different authors and can even lead to wrong conclusions.

The interaction between atoms and the degree of symmetry are also reflected in the excited states. The absorption spectra in the vuv region in condensed heavy rare gases, both in liquid and solid phases, differ from those in gas, exhibiting lines corresponding to Wannier-type "free" excitons instead of the atomic lines. ${ }^{21,22}$ This fact can be taken into account in the dispersion relation, which links the refractive index of the medium with oscillator frequencies. In this paper, we take such approach for calculating the refractive index of condensed xenon. We present a model for the calculation of the refractive index based on the dispersion relation, which incorporates the exciton transition energies. The refractive indices of liquid and solid xenon for different wavelengths are evaluated using this model. Comparison between calculated and experimental values existing in the literature is presented.

\section{CALCULATION OF THE REFRACTIVE INDEX}

The refractive index $\mathbf{n}$ of an isotropic dielectric medium is in general complex and satisfies the dispersion relation ${ }^{23}$

$$
\frac{\mathbf{n}^{2}-1}{\mathbf{n}^{2}+2}=\frac{4 \pi N e^{2}}{3 m} \sum_{i} \frac{f_{i}}{\omega_{i}^{2}-\omega^{2}-i \Gamma_{i} \omega},
$$

where $N$ is the number density of oscillators (atoms or molecules) of the medium, $e$ and $m$ are the charge and mass of the electron, respectively, $f_{i}$ is the oscillator strength for the transition of frequency $\omega_{i}$, and $\Gamma_{i}$ is the width of the line corresponding to $\omega_{i}$. The summation is over all possible transitions. In a wavelength region far from resonance lines, where $\omega_{i}^{2}-\omega^{2}$ is large compared with the term $\Gamma_{i} \omega$, the real part of the refractive index, $n$, can be approximated with

$$
\frac{n^{2}-1}{n^{2}+2} \approx \frac{4 \pi N e^{2}}{3 m} \sum_{i} \frac{f_{i}}{\omega_{i}^{2}-\omega^{2}} .
$$

In case of low-density gases with refractive index close to 1 , Eq. (5) can be simplified to a form which is usually referred to as the Sellmeier equation

$$
n-1 \approx \frac{2 \pi N e^{2}}{m} \sum_{i} \frac{f_{i}}{\omega_{i}^{2}-\omega^{2}} .
$$

This equation was fitted to experimental values of the refractive index of Xe gas at STP $\left(0{ }^{\circ} \mathrm{C}, 760\right.$ torr $)$ between 140 and $254 \mathrm{~nm}$ by Bideau-Mehu et al. $^{24}$ They replaced the complete summation by three terms: two corresponding to the main atomic resonance lines $\lambda_{1}=146.9 \mathrm{~nm}$ and $\lambda_{2}=129.5 \mathrm{~nm}$ (the latter ascribed to the lines at 129.5 and
TABLE I. Energies of the two major exciton lines in liquid and solid xenon estimated from the published reflectivity spectra.

\begin{tabular}{llllr}
\hline \hline & $T(\mathrm{~K})$ & $E_{1}(\mathrm{eV})$ & $E_{2}(\mathrm{eV})$ & \multicolumn{1}{c}{ Ref. } \\
\hline Liquid xenon & 165 & 8.21 & 9.42 & 25 \\
& 163.2 & 8.21 & 9.38 & $5^{\mathrm{a}}$ \\
& 162.5 & 8.18 & 9.36 & 26 \\
& & 8.2 & 9.4 & Average \\
Solid xenon & 161.2 & 8.27 & 9.44 & $5^{\mathrm{a}}$ \\
& 155 & 8.26 & 9.43 & 25 \\
& 137 & 8.28 & 9.45 & 26 \\
& 130 & 8.32 & 9.48 & 25 \\
\hline \hline
\end{tabular}

${ }^{\mathrm{a}}$ For normal incidence; two sets of data were presented in this work; for normal and grazing incidences of the vuv light.

$125.6 \mathrm{~nm}$ ) and the third term accounting for all other transitions. A good agreement with the experimental data was obtained with the equation

$$
\begin{aligned}
n-1= & 1.2055 \times 10^{-2}\left(\frac{0.26783}{46.301-\lambda^{-2}}+\frac{0.29481}{59.578-\lambda^{-2}}\right. \\
& \left.+\frac{5.0333}{112.74-\lambda^{-2}}\right),
\end{aligned}
$$

where $\lambda$ is in micrometers. (Here, we have corrected a printing mistake in the second term in the equation given in Ref. 24).

In order to calculate the refractive index of liquid xenon we used the dispersion relation in the form of Eq. (5) with the summation limited to three terms, as in Ref. 24, and using numerical values for the oscillator strengths and the multiplicative factor on the right side of Eq. (7). However, we substituted the atomic resonance energies. It is well known that the absorption spectra in the condensed xenon differ from those in gas, particularly in the vuv region, exhibiting lines for Wannier-type free excitons instead of the atomic resonance lines. ${ }^{21,22}$ Two exciton lines, $n=1, \Gamma(3 / 2)$ and $n^{\prime}=1, \Gamma(1 / 2)$, were observed in liquid xenon at approximately 8.2 and $9.4 \mathrm{eV}$ (151.2 and $131.9 \mathrm{~nm})$. Table I summarizes the data on the major exciton energies observed in the condensed xenon at various temperatures estimated by us from the original reflectivity spectra published in Refs. 5, 25, and 26. As one can notice from the table, for the liquid state the measurements were done at almost the same temperature and the obtained exciton energies are nearly equal. Hence, we took the average value for each line and arrived at the following equation:

$$
\begin{aligned}
\frac{n^{2}-1}{n^{2}+2}= & 1.2055 \times 10^{-2} \frac{2}{3} \frac{N_{l}}{N_{g}}\left(\frac{0.26783}{43.741-\lambda^{-2}}\right. \\
& \left.+\frac{0.29481}{57.480-\lambda^{-2}}+\frac{5.0333}{112.74-\lambda^{-2}}\right) .
\end{aligned}
$$

In this equation, the factor $2 / 3$ is due to the fact that, opposite Eq. (7), no approximation was done on the left side of the equation. $N_{l} / N_{g}$ is the ratio between the liquid density and that of xenon at STP. In order to be applicable to the liquid or solid state, Eq. (8) should include the effects specific to the condensed phase that are not present in dilute gas. 
TABLE II. Values of density used in this work.

\begin{tabular}{clc}
\hline \hline \multicolumn{1}{c}{ Phase state } & $T(\mathrm{~K})$ & $\rho\left(\mathrm{g} / \mathrm{cm}^{3}\right)$ \\
\hline Gas (STP), Ref. 28 & 273.15 & $5.8984 \times 10^{-3}$ \\
Liquid, Ref. 27 & 170 & 2.9078 \\
& 161.35 & 2.9656 \\
Solid, Ref. 27 & 161.35 & 3.3944 \\
& 150 & 3.4423 \\
& 130 & 3.5137 \\
\hline \hline
\end{tabular}

In fact, some of these effects, such as those due to van der Waals interaction between the atoms, are already taken into account in the ratio $N_{l} / N_{g}$ if the experimental values of density are used (we used values of Refs. 27 and 28). However, the contribution from the terms nonlinear with density may also be important and should be taken into account, in principle, in the "scaling" factor [which is $N_{l} / N_{g}$ in Eq. (8)] and/or in the terms between the parenthesis in Eq. (8). In our approach, we assume that all nonlinear effects of the medium density and phase due to the interaction between the atoms can be taken into account in the oscillator strengths $f_{i}$ and the wavelengths $\lambda_{i}$, which should refer to the excitons, in this case. However, in view of the absence of data on the oscillator strengths associated with the excitons, they were approximated to those reported for the gas phase. ${ }^{24}$ Hence, the sum of the oscillator strengths responsible for $n$ is the same as that for the gas and is 5.5959, which means that only the outer electrons contribute significantly to the refractive index $\left(Z_{\mathrm{Xe}}=54\right)$. It is worth emphasizing that any collective excitations occurring in the condensed phase were not taken into account, but their effect on the refractive index in condensed rare gases is expected to be small. ${ }^{29}$
In order to compare the present model with the existing experimental data, we first calculate the refractive index at $\rho=2.908 \mathrm{~g} / \mathrm{cm}^{3}(T=170 \mathrm{~K})^{27}$ corresponding to the conditions at which $n$ was measured for liquid xenon scintillation light in our previous work. ${ }^{7}$ Taking $^{3} \lambda=0.178 \mu \mathrm{m}$ we obtain $n=1.68$ which is in good agreement with the experimental value $^{7} n=1.69 \pm 0.02$. Concerning the uncertainty associated with the theoretical values, one can present the following considerations. Apart from the uncertainty associated with the approach itself, which is impossible to assess, the main sources of uncertainty in the calculated value of $n$ are the precision with which the wavelengths of the excitons were determined (estimated to be $\approx 8 \times 10^{-4} \mu \mathrm{m}$ ) and the spread of values of the liquid density existing in the literature (estimated to be $\approx 0.3 \%$ ). As a result, the overall uncertainty in the calculated value of $n$ was estimated to be about $0.6 \%$.

Next, we used Eq. (8) to calculate the refractive index of liquid and solid xenon in the vuv and visible regions at the triple point $(T=161.35 \mathrm{~K})$ and at 150 and $130 \mathrm{~K}$, where the experimental data are available. ${ }^{5,9}$ The densities used in our calculation are listed in Table II, while the results for the refractive index are shown in Table III together with the experimental values existing in the literature and calculations made by other authors. For the liquid, the average values of the exciton energies were used, i.e., 8.2 and $9.4 \mathrm{eV}$ (see Table I) since all three experiments were carried out at nearly equal temperatures and the obtained values for the exciton energies are very close. It is worth mentioning that if one takes the exciton energies from Ref. 26, which were obtained at the temperature close to the triple point, the calculated refractive index increases only by about $0.3 \%$ at $178 \mathrm{~nm}$ and $0.05 \%$ in the visible region, which is still in good agreement with the experimental data.

TABLE III. Refractive index of condensed xenon at its scintillation wavelength as well as in the uv and the visible regions.

\begin{tabular}{|c|c|c|c|c|c|c|c|c|c|c|}
\hline \multirow[b]{2}{*}{ State } & \multirow[b]{2}{*}{$T(\mathrm{~K})$} & \multirow{2}{*}{$\begin{array}{l}\text { Experimental } \\
\text { or calculated }\end{array}$} & \multicolumn{7}{|c|}{ Wavelength (nm) } & \multirow[b]{2}{*}{ Ref. } \\
\hline & & & $\mathrm{Xe}^{\mathrm{a}}$ & 188 & 200 & 361.2 & 435.8 & 546.1 & 643.9 & \\
\hline \multirow[t]{8}{*}{ Liquid } & 170 & Expt. & 1.69 & & & & & & & 7 \\
\hline & & Calc. & 1.68 & & & & & & & p.w. ${ }^{b}$ \\
\hline & 163.2 & Expt. & 1.66 & 1.60 & 1.56 & & & & & 5 \\
\hline & 161.35 (TP) & Expt. & 1.5655 & & & & & & & 6 \\
\hline & & Expt. & & & & 1.4111 & 1.4001 & 1.3918 & 1.3876 & 9 \\
\hline & & Calc. & 1.70 & 1.62 & 1.566 & 1.4094 & 1.3958 & 1.3857 & 1.3810 & p.w. ${ }^{b}$ \\
\hline & & Calc. & & & & 1.4137 & 1.4019 & 1.3934 & 1.3896 & $9^{c}$ \\
\hline & & Calc. & 1.59 & & & & & & & 32 \\
\hline \multirow[t]{7}{*}{ Solid } & 161.2 & Expt. & 1.79 & 1.69 & 1.65 & & & & & 5 \\
\hline & 161.35 (TP) & Expt. & & & & 1.4747 & 1.4608 & 1.4507 & 1.4461 & 9 \\
\hline & 161.35 (TP) & Calc. & 1.90 & 1.73 & 1.662 & 1.4765 & 1.4603 & 1.4484 & 1.4427 & p.w. ${ }^{d}$ \\
\hline & 150 & Expt. & & & & 1.4805 & 1.4667 & 1.4566 & 1.4520 & 9 \\
\hline & & Calc. & 1.93 & & & 1.4847 & 1.4682 & 1.4560 & 1.4502 & p.w. ${ }^{\mathrm{e}}$ \\
\hline & 130 & Expt. & & & & 1.4901 & 1.4763 & 1.4662 & 1.4616 & 9 \\
\hline & & Calc. & 1.93 & & & 1.4946 & 1.4778 & 1.4654 & 1.4596 & p.w. ${ }^{\mathrm{f}}$ \\
\hline
\end{tabular}

\footnotetext{
Xenon scintillation: $\lambda=178 \mathrm{~nm}$ (for liquid) and $\lambda=172 \mathrm{~nm}$ (for solid) from Ref. 3 .

${ }^{\mathrm{b}}$ The average value for each exciton energy was used (see Table I).

${ }^{c}$ Values calculated using the tight-binding exciton model with the parameters listed in Table VII of Ref. 9.

${ }^{\mathrm{d}}$ Exciton energies at $161.2 \mathrm{~K}$ from Ref. 5 .

${ }^{\mathrm{e}}$ Exciton energies at $155 \mathrm{~K}$ from Ref. 25.

${ }^{\mathrm{f}}$ Exciton energies at $130 \mathrm{~K}$ from Ref. 25.
} 


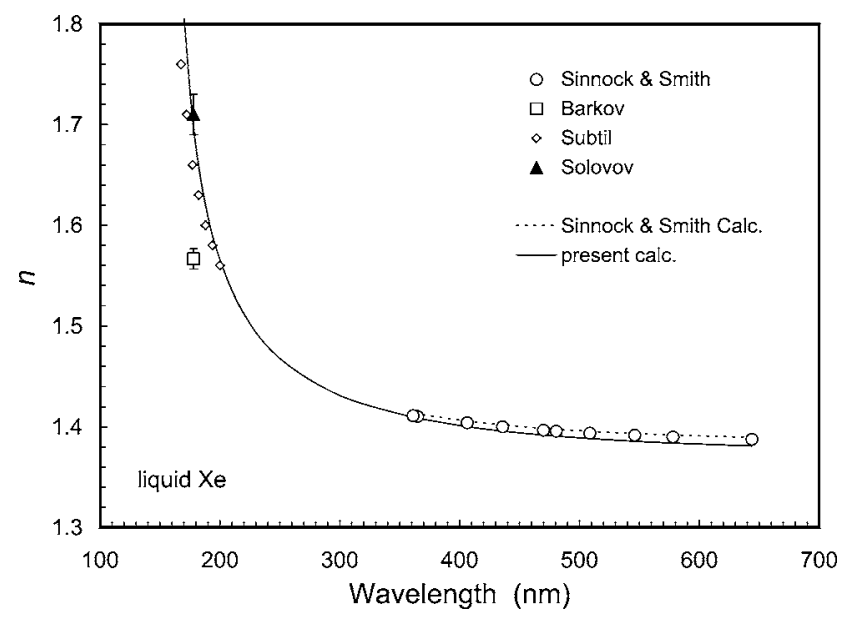

FIG. 1. The refractive index of liquid xenon at the triple point $(T=161.35 \mathrm{~K})$ as a function of wavelength. Experimental data: circles, Ref. 9; square, Ref. 6; diamonds, Ref. 5; and triangle, Ref. 7. The last value was measured at $T=170 \mathrm{~K}$ and recalculated for triple point, as explained in the text. The solid line was calculated with the approach proposed in the present work. The broken line was calculated using the tight-binding exciton model, in the range where it is valid, with the parameters reported in Table VII of Ref. 9.

For solid xenon, the results shown in Table III were calculated with the exciton energies from Table I at the temperature closest to that of the respective experimental data set as indicated in Table III.

\section{DISCUSSION}

In the visible region of the spectrum, a fairly good agreement has been found between the experimental values of the refractive index of condensed rare gases and the predictions based on the tight-binding exciton model, which is valid only at frequencies $\omega \ll \omega_{0}$ where $\omega_{0}$ are the natural frequencies of the oscillators. ${ }^{9,30}$ When $\omega$ approaches the resonance frequencies, the use of the dispersion relation is more adequate, as it is done in the present work.

A significant part of the existing experimental data for the refractive index of liquid xenon is obtained at the temperature near the triple point $(T=161.35 \mathrm{~K})$. These data are presented in Fig. 1 along with the calculations with the tightbinding exciton model from Ref. 9 in the visible region (dashed line) and with our model (solid line). For the sake of comparison, our experimental value for xenon scintillation light $^{7}$ measured at $T=170 \mathrm{~K}$ was scaled to the temperature of triple point using the density dependence from Eq. (8) and the density values listed in Table I.

For the liquid xenon scintillation light, the refractive index of liquid xenon calculated in this work is in good agreement with the experimental value reported: ${ }^{7}$ the experiment at $T=170 \mathrm{~K}$ gives $n=1.69 \pm 0.02$, while the calculation results in $n=1.68$ (see Table III). Practically the same value of $n$, differing only by $0.05 \%$, is obtained if, instead of Eq. (7) adopted from Ref. 24, one uses the empirical dispersion equation from Ref. 31 with a similar replacement of the two atomic lines by the exciton frequencies and density "scaling" to the liquid. It is also worth mentioning that if Eq. (8) is used with atomic frequencies (and not with those corre- sponding to excitons, as it is done in the present work), it results in a much lower value of the refractive index, $n=1.64$ for $170 \mathrm{~K}$.

Comparing our calculation with the data from Ref. 5 measured with the liquid near the triple point $(163.2 \mathrm{~K})$ in the wavelength region from 200 down to $153.3 \mathrm{~nm}$, we also find the agreement to be quite good although the uncertainty in $n$ is not mentioned in Ref. 5. Thus, the measured value of the refractive index at $\lambda=177 \mathrm{~nm}$ is 1.66 , while we calculate 1.70 , for this temperature. For $\lambda=188 \mathrm{~nm}$ and $\lambda=200 \mathrm{~nm}$ the values from Ref. 5 are 1.60 and 1.56 , while our calculation gives 1.62 and 1.556, respectively.

Regarding the value of $n$ for liquid xenon at triple point and for $\lambda=180 \mathrm{~nm}$ presented in Ref. 6 (the authors quote $n=1.5655 \pm 0.0024 \pm 0.0078)$, it is well below any of the calculated values mentioned above. Regrettably, that paper does not provide enough details to allow discussion of possible reasons for the discrepancy. A similar value, $n=1.59$ at $\lambda=175 \mathrm{~nm}$, was obtained earlier by Braem et al. ${ }^{32}$ from the experimental data of Ref. 9 at $\lambda>350 \mathrm{~nm}$ by extrapolation to the vuv region with a modified form of the LorentzLorenz equation (not specified in the paper). However, the authors stressed that the extrapolation is very sensitive to the functional form of the extrapolation and to small changes in the values of the refractive index at longer wavelengths.

A value of the refractive index of $n=1.6733$, measured at $\lambda=176 \mathrm{~nm}$ in liquid xenon presumably at the temperature near the triple point (the temperature is not indicated in the communication), can also be referred to ${ }^{33}$ in support of our calculations and previous measurements. ${ }^{7}$

When we use our approach in the long wavelength region from 361 to $644 \mathrm{~nm}$, a reasonable agreement is still found between the calculated and experimental values (see Table III and Fig. 1). The difference between them never exceeds $0.5 \%$. However, it should be noted that the oscillator strengths and the multiplicative constant used in Eq. (8) were originally $^{24}$ obtained by fitting the Sellmeier formula [our Eq. (6)] to the data taken at $\lambda$ between 140 and $254 \mathrm{~nm}$, thus its use outside this wavelength range is not entirely reliable.

Concerning our estimates for solid xenon, in the visible region they agree moderately well with the experimental data available $^{9}$ for all three temperatures $(130 \mathrm{~K}, 150 \mathrm{~K}$, and near the triple point, see Table III). The deviations vary from about $0.3 \%$ to $0.05 \%$, while the uncertainty in the experimental values of the refractive index is quoted to be about $0.1 \%$. As for the vuv region, the agreement is not so good. Thus, the values of $1.79,1.69$, and 1.65 were measured for $172 \mathrm{~nm}$ (this wavelength corresponds to solid xenon emission), 188, and $200 \mathrm{~nm}$, respectively, at $161.2 \mathrm{~K}$ (systematic errors were not clearly specified $\left.{ }^{5}\right)$. Our calculation at the temperature of triple point $(161.35 \mathrm{~K})$ gives the respective indices of 1.90, 1.73, and 1.66. Compared with the liquid state, the disagreement at short wavelengths in the solid can be understood if one takes into account the fact that in solid xenon the wavelength of the scintillation emission is closer to the first exciton line than it is in the liquid. Solid xenon scintillates $^{5}$ at $\lambda=172 \mathrm{~nm}$ and the first exciton band is at about $\lambda_{\mathrm{ex}}=150 \mathrm{~nm}$, while in the liquid the respective wavelengths are $\lambda=178 \mathrm{~nm}$ and $\lambda_{\mathrm{ex}}=151 \mathrm{~nm}$ (see Table I). 


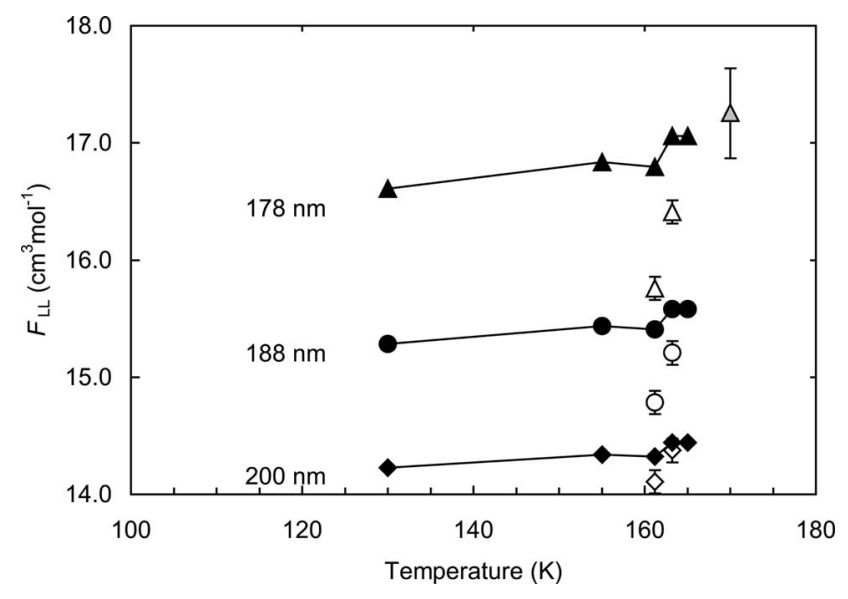

FIG. 2. Lorentz-Lorenz function $F_{\mathrm{LL}}$ as a function of temperature in the vuv region. Open symbols correspond to $F_{\text {LL }}$ obtained using the definition of the Lorentz-Lorenz function [i.e., Eq. (2)] and the experimental values of $n$ reported in Subtil et al. (Ref. 5), while a grey triangle corresponds to that obtained with the refractive index from Solovov et al. (Ref. 7). The filled symbols, connected by solid lines: calculated with the right side of Eq. (8) and the densities listed in Table II (the lines are for eye guidance only).

A relevant issue related to the refractive index that has been discussed in the literature is whether the LorentzLorenz function $F_{\mathrm{LL}}$, defined by Eq. (2), depends on the density and phase of the medium. ${ }^{8,9,11,30}$ Our method allows us to compute $F_{\mathrm{LL}}$ for different densities and phase states of the condensed xenon, provided that the exciton lines are known. A gradual shift in the exciton energies to higher energies with increase of density is clearly seen from Table I, although the uncertainty, estimated to be about 0.05 to $0.02 \mathrm{eV}$, is rather large. Using these energies in Eq. (8) one can obtain $F_{\text {LL }}$ (Figs. 2 and 3). The result shows that $F_{\text {LL }}$ decreases with decreasing temperature in the whole wavelength region from 643.9 down to $178 \mathrm{~nm}$, the effect being more significant for shorter wavelengths.

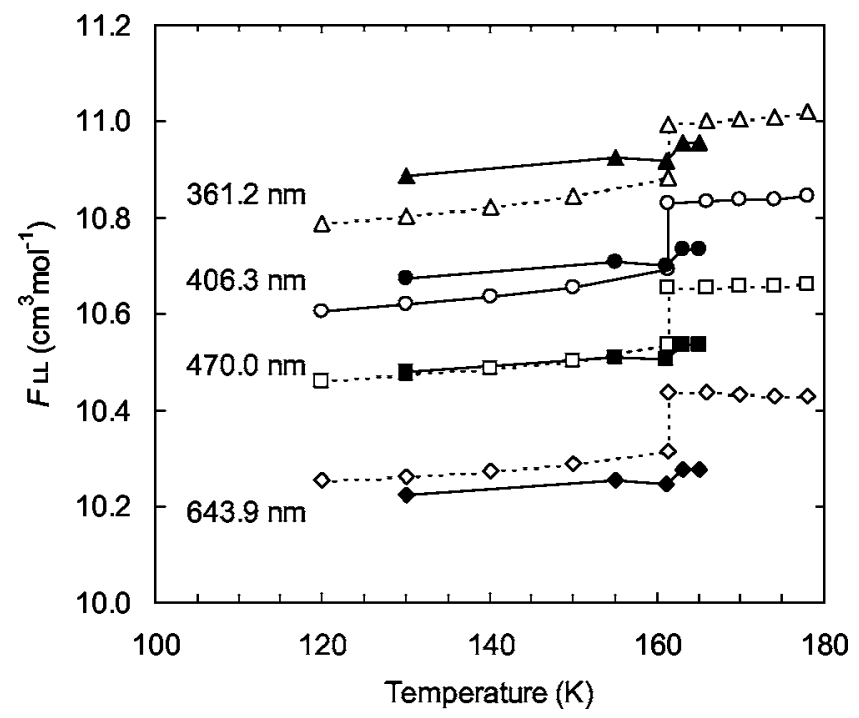

FIG. 3. Lorentz-Lorenz function $F_{\mathrm{LL}}$ as a function of temperature in the visible region. Open symbols connected by broken lines correspond to $F_{\text {LL }}$ obtained using the definition of the Lorentz-Lorenz function [i.e., Eq. (2)] and the experimental values of $n$ from Sinnock and Smith (Ref. 9). The filled symbols, connected by solid lines: calculated with the right side of Eq. (8) and the densities listed in Table II (the lines are for eye guidance only).
The Lorentz-Lorenz function obtained with the dispersion relation can be compared with $F_{\text {LL }}$ calculated using its definition [Eq. (2)] and the experimental values of the refractive index of liquid and solid xenon. ${ }^{9,30}$ As one can see from Figs. 2 and 3, both methods result in a similar tendency with the temperature in the solid state as well as at the solid-liquid transition point. In the case of our model, that tendency reflects a trend in exciton energies with temperature and phase. It is preserved in spite of the uncertainty in $F_{\mathrm{LL}}$ due to the imprecision in the exciton energies being rather large. The uncertainty in $F_{\mathrm{LL}}$ is estimated to be of the order of 0.02 and $0.1 \mathrm{~cm}^{3} \mathrm{~mol}^{-1}$ for the visible region and for the vuv region, respectively. The sharp decrease of $F_{\mathrm{LL}}$ on freezing was also referred in the vuv region by Subtil et al. ${ }^{5}$

The jump of $F_{\text {LL }}$ at the phase-transition temperature is, however, more pronounced when calculated with the experimental values of the refractive index (open symbols in Figs. 2 and 3). For the wavelengths of 200, 188, and $178 \mathrm{~nm}$, it is about $1.9 \%, 2.9 \%$, and $4.1 \%$, respectively. If calculated with Eq. (8), the respective values are $0.9 \%, 1.1 \%$, and $1.6 \%$. The decrease of $F_{\text {LL }}$ on liquid freezing and its further decrease with decreasing temperature of the solid have been attributed to the high symmetry of crystal, for which, unlike the liquid, the contribution from the configuration terms is negligible. ${ }^{9}$ In the present model, this effect is expressed as a change in the exciton energies.

Altogether, a good agreement with the experimental data on the refractive index of the condensed xenon has been obtained with the theoretical approach proposed in this work. Nevertheless, it can be substantially improved. In the present approach, the correlations between atoms are taken into account only by using for Eq. (5) the wavelengths of exciton lines instead of those of the atomic lines observed in the gas phase. However, due to the lack of information on the oscillator strengths of the transitions in the condensed phase, they were assumed to be the same as in the gas phase. The oscillator strengths of the two main excitons were approximated to those of the two main atomic lines observed at similar wavelengths. Furthermore, due to the formation of the conduction bands, the ionization potential in the condensed rare gases is known to be lower than in the gas phase. ${ }^{21,26}$ Hence, a change in the distribution of the oscillator strengths associated with the transitions to the conduction band can be expected. This was not taken into account in the present evaluations.

A more precise knowledge of the exciton energies for different temperatures and phase states is also required. It is especially important for the vuv part of the spectrum, at wavelengths approaching the resonance lines. The relative contribution of the first two terms in Eq. (8) significantly increases at $178 \mathrm{~nm}$ as compared, for example, with that at $546.1 \mathrm{~nm}$, the ratio of the three terms being equal to $0.231: 0.119: 0.650$ and $0.114: 0.094: 0.792$, respectively (the sum is normalized to 1 ).

\section{CONCLUSIONS}

The refractive index of liquid xenon at the wavelength of its scintillation light was calculated to be $n=1.68$ at $170 \mathrm{~K}$ 
(1.70 at the triple point) using the dispersion relation combined with the free-exciton model. This approach was also able to reproduce fairly well the published experimental values of the refractive index of liquid and solid xenon in the vuv and visible range. Moreover, it led to a trend of the dependence of the Lorentz-Lorenz function with temperature and phase similar to that reported in literature. $F_{\mathrm{LL}}$ decreases sharply on phase transition from liquid to solid, further decreasing with increasing density of the solid. However, for a satisfactory test of this model, more measurements of the reflective index in the condensed phase are required, in the uv region in particular. Moreover, in order to improve the model, more data on the oscillator strengths and the exciton energies in the condensed xenon are needed. It is expected that the recent advances in synchrotron facilities can make it possible to obtain information on the oscillator strengths.

\section{ACKNOWLEDGMENTS}

We express our gratitude to Professor Y. Hatano for valuable discussions and comments. This work was financed by Project No. CERN/FNU/43729/2001 from the Fundação para a Ciência e Tecnologia, Portugal. One of the authors (V.S.) was supported by a fellowship, PRAXIS (XXI/BD/ 3892/96) from the same organization. Another author (A.H.) received a fellowship from Fundação Oriente, Portugal.

${ }^{1}$ T. Doke and K. Masuda, Nucl. Instrum. Methods Phys. Res. A 420, 62 (1999).

${ }^{2}$ M. I. Lopes and V. Chepel, in Electronic Excitations in Liquefied Rare Gases, edited by W. F. Schmidt and E. Illenberger (American Scientific, North Lewis Way, CA, 2005), pp. 331-388; IEEE Trans. Dielectr. Electr. Insul. 10, 994 (2003).

${ }^{3}$ J. Jortner, L. Meyer, S. A. Rice, and E. G. Wilson, J. Chem. Phys. 42, 4250 (1965)

${ }^{4}$ N. Schwentner, E. E. Koch, and J. Jortner, Electronic Excitations in Condensed Rare Gases, Springer Tracts in Modern Physics Vol. 107 (Springer, Berlin, 1985).

${ }^{5}$ J. L. Subtil, P. Laporte, R. Reininger, and V. Saile, Phys. Status Solidi B 143, 783 (1987). The values of the refractive index $\left(n_{L}\right)$, calculated by extrapolation of the dilute gas data with the Lorentz-Lorenz formula (Table I of this reference), are not correct because of a printing mistake in Ref. 24.
${ }^{6}$ L. M. Barkov, A. A. Grebenuk, N. M. Ryskulov, P. Yu. Stepanov, and S. G. Zverev, Nucl. Instrum. Methods Phys. Res. A 379, 482 (1996).

${ }^{7}$ V. N. Solovov, V. Chepel, M. I. Lopes, A. Hitachi, R. Ferreira Marques, and A. J. P. L. Policarpo, Nucl. Instrum. Methods Phys. Res. A 516, 462 (2004).

${ }^{8}$ J. A. Chapman, P. C. Finnimore, and B. L. Smith, Phys. Rev. Lett. 21, 1306 (1968)

${ }^{9}$ A. C. Sinnock and B. L. Smith, Phys. Rev. 181, 1297 (1969).

${ }^{10}$ R. F. Guertin and F. Stern, Phys. Rev. 134, A427 (1964).

${ }^{11}$ D. H. Garside, H. V. Mølgaard, and B. L. Smith, J. Phys. B 1, 449 (1968).

${ }^{12}$ C. P. Abbiss, C. M. Knobler, R. K. Teague, and C. J. Pings, J. Chem. Phys. 42, 4145 (1965).

${ }^{13}$ A. Michels, C. A. ten Seldam, and S. D. J. Overdijk, Physica (Amsterdam) 17, 781 (1951)

${ }^{14}$ R. L. Amey and R. H. Cole, J. Chem. Phys. 40, 146 (1964).

${ }^{15}$ A. D. Buckingham, Trans. Faraday Soc. 52, 1035 (1956); A. D. Buckingham, R. H. Cole, and H. Sutter, J. Chem. Phys. 52, 5960 (1970).

${ }^{16}$ H. J. Achtermann, J. G. Hong, G. Magnus, R. A. Aziz, and M. J. Slaman, J. Chem. Phys. 98, 2308 (1993).

${ }^{17}$ J. G. Kirkwood, J. Chem. Phys. 4, 592 (1936).

${ }^{18}$ C. A. ten Seldam and S. R. de Groot, Physica (Amsterdam) 18, 910 (1952).

${ }^{19}$ L. Jansen and P. Mazur, Physica (Amsterdam) 21, 193 (1955).

${ }^{20}$ R. Yaris and B. Kirtman, J. Chem. Phys. 37, 1775 (1962).

${ }^{21}$ G. Baldini, Phys. Rev. 128, 1562 (1962).

${ }^{22}$ I. T. Steinberger and O. Schnepp, Solid State Commun. 5, 417 (1967).

${ }^{23}$ R. W. Ditchburn, Light (Blackie \& Son, London, 1963).

${ }^{24}$ A. Bideau-Mehu, Y. Guern, R. Abjean, and A. Johannin-Gilles, J. Quant. Spectrosc. Radiat. Transf. 25, 395 (1981). There is a printing mistake in this reference: the value of $\lambda_{2}^{-2}$ in the equation in page 401 should be 59.578 instead of 50.578 .

${ }^{25}$ D. Beaglehole, Phys. Rev. Lett. 15, 551 (1965).

${ }^{26}$ U. Asaf and I. T. Steinberger, Phys. Lett. 34, 207 (1971).

${ }^{27}$ V. A. Rabinovich, A. A. Vasserman, V. I. Nedostup, and L. S. Veksler, Thermophysical Properties of Neon, Argon, Krypton, and Xenon (Hemisphere, Washington, 1988).

${ }^{28}$ P. J. Linstrom and W. G. Mallard, Eds. NIST Chemistry WebBook, NIST Standard Reference Database No. 69, edited by P. J. Linstrom and W. G. Mallard (National Institute of Standards and Technology, Gaithersburg, MD, 2001) (http://webbook.nist.gov/chemistry).

${ }^{29}$ Y. Hatano and M. Inokuti, in Atomic and Molecular Data for Radiotherapy and Radiation Research, edited by M. Inokuti (IAEA, Vienna, 1995), Chap. 5.

${ }^{30}$ A. C. Sinnock, J. Phys. C 13, 2375 (1980).

${ }^{31}$ P. J. Leonard, At. Data Nucl. Data Tables 14, 21 (1974).

${ }^{32}$ A. Braem et al., Nucl. Instrum. Methods Phys. Res. A 320, 228 (1992).

${ }^{33}$ J. Seguinot and T. Ypsilantis (unpublished). 
The Journal of Chemical Physics is copyrighted by the American Institute of Physics (AIP). Redistribution of journal material is subject to the AIP online journal license and/or AIP copyright. For more information, see http://ojps.aip.org/jcpo/jcper/jsp 